\title{
Oleanolic Acid Provides Neuroprotection against Ischemic Stroke through the Inhibition of Microglial Activation and NLRP3 Inflammasome Activation
}

\author{
Arjun Sapkota and Ji Woong Choi* \\ Laboratory of Neuropharmacology, College of Pharmacy and Gachon Institute of Pharmaceutical Sciences, Gachon University, \\ Incheon 21936, Republic of Korea
}

\begin{abstract}
Oleanolic acid (OA), a natural pentacyclic triterpenoid, has been reported to exert protective effects against several neurological diseases through its anti-oxidative and anti-inflammatory activities. The goal of the present study was to evaluate the therapeutic potential of OA against acute and chronic brain injuries after ischemic stroke using a mouse model of transient middle cerebral artery occlusion (tMCAO, MCAO/reperfusion). OA administration immediately after reperfusion significantly attenuated acute brain injuries including brain infarction, functional neurological deficits, and neuronal apoptosis. Moreover, delayed administration of OA (at $3 \mathrm{~h}$ after reperfusion) attenuated brain infarction and improved functional neurological deficits during the acute phase. Such neuroprotective effects were associated with attenuation of microglial activation and lipid peroxidation in the injured brain after the tMCAO challenge. OA also attenuated NLRP3 inflammasome activation in activated microglia during the acute phase. In addition, daily administration of OA for 7 days starting from either immediately after reperfusion or 1 day after reperfusion significantly improved functional neurological deficits and attenuated brain tissue loss up to 21 days after the tMCAO challenge; these findings supported therapeutic effects of OA against ischemic stroke-induced chronic brain injury. Together, these findings showed that OA exerted neuroprotective effects against both acute and chronic brain injuries after tMCAO challenge, suggesting that OA is a potential therapeutic agent to treat ischemic stroke.
\end{abstract}

Key Words: Oleanolic acid, Ischemic stroke, Microglia, Lipid peroxidation, NLRP3 inflammasome activation

\section{INTRODUCTION}

Oleanolic acid (3ß-hydroxyolean-12-en-28-oic acid, OA) is a natural compound of pentacyclic triterpenoid compound widely spread in a variety of plant species, especially in plants belonging to the Oleaceae family (Ayeleso et al., 2017). In China, for a long time, OA has been clinically used to treat hepatitis (Liu, 2005; Xu et al., 2014). Consequently, there has been growing research interest in $\mathrm{OA}$ and its derivatives, and it has also been reported to have multiple pharmacological effects including anti-inflammatory, anti-oxidative, anti-microbial, anticancer, hepatoprotective, anti-diabetic, and cardioprotective effects (Castellano et al., 2013; Martin et al., 2014; Ayeleso et al., 2017; Ziberna et al., 2017; Niu et al., 2018; Wang et al., 2018b). Moreover, OA can attenuate brain damage in several central nervous system (CNS) diseases including ischemic stroke (Rong et al., 2011; Shi et al., 2021), hypoxia (Caltana et al., 2014), multiple sclerosis (Martin et al., 2010), spinal cord injury (Wang et al., 2020), and Alzheimer's disease (Wang et al., 2018a; Gudoityte et al., 2021). In particular, OA has been suggested as a neuroprotective agent against ischemic stroke since it can prevent brain damage, such as brain infarction, neurological dysfunction, oxidative stress, and blood-brain barrier disruption, after transient middle cerebral artery occlusion (tMCAO) (Rong et al., 2011; Shi et al., 2021). However, it is unclear whether OA can exert therapeutic effects against ischemic stroke since such previous studies have demonstrated its prophylactic effects by administrating OA prior to the stroke challenge. In addition, there was no studies on whether neuroprotective effects of OA against ischemic stroke could be preserved by delayed administration (i.e., several $h$ after stroke) despite such evaluation being an ideal criterion for judging its therapeutic potential. Therefore, the goal of the present study was to evaluate the therapeutic potential of OA

\section{Open Access https://doi.org/10.4062/biomolther.2021.154}

This is an Open Access article distributed under the terms of the Creative Commons Attribution Non-Commercial License (http://creativecommons.org/licenses/by-nc/4.0/) which permits unrestricted non-commercial use, distribution, and reproduction in any medium, provided the original work is properly cited.
Received Oct 6, 2021 Revised Nov 13, 2021 Accepted Nov 15, 2021 Published Online Dec 7, 2021

\section{*Corresponding Author}

E-mail: pharmchoi@gachon.ac.kr Tel: +82-32-820-4955, Fax: +82-32-820-4829 
neuroprotective effects against ischemic stroke, and to demonstrate the associated mechanisms of action.

In the present study, the tMCAO challenge (90 min of occlusion and reperfusion) was used as an ischemic stroke challenge in mice and neuroprotective effects of OA were determined during acute ( 1 and/or 3 days after tMCAO challenge) and chronic (up to 21 days after tMCAO challenge) ischemic injuries. First, the therapeutic potential of OA effects against ischemic stroke during the acute phase was evaluated by assessing its effects on brain damages when administered immediately after reperfusion. Next, OA was administered $3 \mathrm{~h}$ after reperfusion and its effects on brain damages were assessed to determine whether delayed administration of OA could also exert similar therapeutic effects during the acute phase. Further, to demonstrate underlying mechanisms, the OA ability to attenuate microglial activation, lipid peroxidation, and NLRP3 inflammasome activation during the acute phase was also evaluated. The therapeutic potential of OA effects against ischemic stroke during the chronic phase (up to 21 days after tMCAO challenge) was determined by assessing its effects on brain damages when administered either immediately after reperfusion or 1 day after reperfusion.

\section{MATERIALS AND METHODS}

\section{Animal}

Male ICR mice (6 weeks old) were obtained from Orient Bio (Seongnam, Korea). All animal experiments and handling were performed under the Animal Care and Use Guidelines provided by the Gachon University (Incheon, Korea). All animal experiments were approved by Gachon Institutional Animal Care and Use Committee (GIACUC) (approved animal experiment: GIACUC-R2021012). The mice were kept under environmental controlled conditions; temperature $\left(22 \pm 2^{\circ} \mathrm{C}\right)$, relative humidity ( $60 \pm 10 \%$ ), and light cycle (a $12 \mathrm{~h}$ dark/light). Mice had free access to food and water ad libitum.

\section{tMCAO challenge}

Transient middle cerebral artery occlusion (tMCAO) was done using the intraluminal suture occlusion method as described previously (Gaire et al., 2020). Briefly, the mice were anesthetized with isoflurane with an air mixture of $\mathrm{O}_{2}: \mathrm{N}_{2} \mathrm{O}$ (1:3 ratio) ( $3 \%$ for induction and $1.5 \%$ for maintenance). A ventral neck incision was made and the right common carotid artery (CCA) was carefully separated from the vagus nerve. The middle cerebral artery (MCA) was occluded by inserting silicone-coated monofilament $(5-0,9 \mathrm{~mm})$ towards the MCA from internal CCA bifurcation. Reperfusion was achieved by withdrawing the monofilament after 90 min of the occlusion to restore the blood flow. The same surgical procedures were applied for sham group mice except for MCA occlusion. The mice body temperature was maintained at $37^{\circ} \mathrm{C}$ throughout the experiment. To reduce post-surgery pain, $2 \%$ lidocaine gel was applied to the surgical area.

\section{OA administration}

Prior to reperfusion, mice were randomly divided into a vehicle (10\% Tween-80)- or an OA-administered group.

To determine the protective effects of OA against acute brain injury following ischemic stroke, a vehicle or OA $(1,3$, 10, and 30 mg/kg, p.o.; Sigma-Aldrich, St. Louis, MO, USA) was administered to mice immediately after reperfusion. Alternatively, OA was administered to mice at $3 \mathrm{~h}$ after reperfusion for the time window experiment.

To determine the protective effects of OA against chronic brain injury following ischemic stroke, OA was administered once per day for 7 days starting either immediately after reperfusion for the immediate administration group or at 1 day after reperfusion for the delayed administration group.

\section{Assessment of functional neurological deficits}

Modified neurological severity score (mNSS) scale was used to assess the functional neurological deficits in motor, sensory, reflex, and balance functions every day up to 21 days, as previously described (Gaire et al., 2020). The mNSS ranged from 0 to 18 points ( 0 for normal and 18 for maximum deficit score).

\section{Determination of infarction volume}

The mice were sacrificed with $\mathrm{CO}_{2}$ exposure and their brains were collected to determine the infarction volume through 2\% 2,3,5-triphenyltetrazolium chloride (TTC) staining 1 day after tMCAO challenge. Brains were sliced into $2 \mathrm{~mm}-$ thick coronal sections and stained with $2 \%$ TTC in saline at $37^{\circ} \mathrm{C}$, as previously described (Gaire et al., 2020). The stained slices were photographed and the infarction area of each slice were analyzed using ImageJ software (National Institutes of Health, Bethesda, MD, USA).

\section{Histological analysis}

Preparation of tissue samples: Brain samples were harvested 1 and 3 days after the tMCAO challenge. The mice were anesthetized with isoflurane, perfused transcardially with ice-cold phosphate-buffered saline (PBS), and fixed with $4 \%$ paraformaldehyde (PFA). For immunohistochemical analysis, the brains were collected and post-fixed with 4\% PFA for an additional $24 \mathrm{~h}$, transferred into $30 \%$ sucrose solution for cryoprotection, and embedded in Tissue-Tek Optimal Cutting Temperature compound and coronally sectioned into $20 \mu \mathrm{m}$ thick sections using a cryostat machine (RD-2230, Roundfin, Shenyang, Liaoning, China).

TUNEL assay: Neuronal apoptosis in injured brains was analyzed by TUNEL assay using In Situ Cell Death Detection Kit (Roche, Mannheim, Germany) 1 day after the tMCAO challenge, as described previously (Sapkota et al., 2020). Brain sections were post-fixed with $4 \%$ PFA, treated with $50 \mathrm{mM}$ ammonium chloride, exposed to $1 \% \mathrm{H}_{2} \mathrm{O}_{2}$ in $1 \% \mathrm{NaOH}$, exposed to $0.03 \%$ glycine, and blocked with $1 \%$ fetal bovine serum (FBS) in PBS containing $0.3 \%$ Triton $X-100$. Brain sections were incubated with mouse anti-NeuN (1:200, Millipore, Burlington, MA, USA) overnight at $4^{\circ} \mathrm{C}$ and labeled with AF488-conjugated secondary antibody $(1: 1,000$, Jackson ImmunoResearch, West Grove, PA, USA) for $2 \mathrm{~h}$ at room temperature $(R / T)$. The sections were then washed with PBS and labeled with a mixture of Enzyme solution and Label solution for $1 \mathrm{~h}$ at R/T. These sections were washed with PBS and distilled water and then mounted with VECTASHIELD ${ }^{\circledR}$ mounting media (Vector Laboratories, Burlingame, CA, USA).

Iba1 and 4- hydroxynonenal (4-HNE) immunohistochemistry: Microglial activation and lipid peroxidation were determined by immunohistochemical analysis, as previously described (Gaire et al., 2020). Briefly, the brain sections were post-fixed with $4 \%$ PFA, washed with PBS, treated with $\mathrm{H}_{2} \mathrm{O}_{2}$, 
blocked with $1 \%$ FBS in PBS, and incubated with rabbit primary antibody anti-lba1 (1:500, Wako Pure Chemicals, Chou-ku, Osaka, Japan) or 4-HNE (1:500, Bioss, Freiburg, Germany) overnight at $4^{\circ} \mathrm{C}$. The sections were then labeled with a biotinylated secondary antibody $(1: 200$, Santa Cruz Biotechnology, Dallas, TX, USA) and incubated with $A B C$ reagent $(1: 100$, Vector Laboratories). Iba1 or 4-HNE signals were visualized by incubating the sections with a solution containing 3,3'-diaminobenzidine substrate (Dako, Santa Clara, CA, USA). The brain samples were washed with water, dehydrated with alcohol series and xylene, and mounted with Entellan media (Merck, Darmstadt, Germany).

NLRP3/Iba1 or NLRP3/ASC double immunofluorescence: The brain sections were post-fixed in $4 \%$ PFA, treated with $0.01 \mathrm{M}$ sodium citrate at $90-100^{\circ} \mathrm{C}$, exposed to $1 \% \mathrm{H}_{2} \mathrm{O}_{2}$, and blocked with $1 \%$ FBS. For NLRP3/lba1 double immunofluorescence, the brain sections were incubated with mouse antiNLRP3 (1:100, AdipoGen Life Sciences, San Diego, CA, USA) and rabbit anti-lba1 (1:500, Wako Pure Chemicals) primary antibodies overnight at $4^{\circ} \mathrm{C}$. For NLRP3/ASC double immunofluorescence, the sections were incubated with mouse antiNLRP3 (1:100) and rabbit anti-ASC (1:200, AdipoGen Life Sciences) primary antibodies. The brain sections were then labeled with AF488- and Cy3-conjugated secondary antibodies $(1: 1,000$, Jackson ImmunoResearch) for $2 \mathrm{~h}$ at $\mathrm{R} / \mathrm{T}$. The sections were counterstained with 4',6-diamidino-2-phenylindole (DAPI) (Carl Roth, Karlsruhe, Germany) and mounted with VECTASHIELD ${ }^{\circledR}$ (Vector Laboratories).

Image preparation and quantification: Brain images were photographed with a microscope equipped with a DP72 camera (BX53T, Olympus, Shinjuku-ku, Tokyo, Japan) or with a confocal microscope (Eclipse A1 Plus, Nikon, Minato City, Tokyo, Japan). All the representative images were prepared using Adobe Photoshop Element 8 (Adobe, San Jose, CA, USA). Three different images $(200 \mu \mathrm{m} \times 200 \mu \mathrm{m})$ of each brain region were used to quantify the immunopositive cells. The number of immunopositive cells was counted manually and converted to the number of cells per unit area. In the case of ASC/NLRP3 double immunofluorescence, the numbers of ASC/NLRP3-double immunopositive cells of each image were manually counted and the values were expressed by the percentage of the number of cells with ASC/NLRP3 speck versus the total number of cells (DAPI-positive cells).

\section{Assessment of brain tissue loss}

Brain samples were harvested 21 days after the tMCAO challenge. The mice were anesthetized with isoflurane, perfused transcardially with ice-cold PBS, and fixed with 4\% PFA. The collected brains were then photographed. The percentage of the brain tissue loss was measured using ImageJ software (National Institutes of Health) with the following formula: (contralateral hemisphere area - ipsilateral area)/contralateral hemisphere area $\times 100 \%$.

\section{Statistical analysis}

Data were expressed as mean \pm standard error of mean (SEM). Statistical analysis was performed using GraphPad Prism 7 (GraphPad Software Inc., La Jolla, CA, USA). Statistical differences between the two groups were analyzed by Student's $t$-test. Statistical difference among multiple groups were analyzed by one-way ANOVA followed by NewmanKeuls test. Statistical significance was set at $p<0.05$.

\section{RESULTS}

\section{$O A$ attenuates acute brain injury after $\mathrm{MCAO}$ challenge}

We first evaluated whether a therapeutic regimen of $O A$ could attenuate acute brain injury after tMCAO challenge when administered immediately after reperfusion. The effects were evaluated in a dose-dependent manner $(3,10$, and 30 $\mathrm{mg} / \mathrm{kg}$ ). The protective effects of OA were assessed by measuring brain infarction and mNSS 1 day after the tMCAO challenge. OA administration immediately after reperfusion significantly reduced brain infarction and improved neurological functions in a dose-dependent manner as compared with the vehicle-administered group (Fig. 1A-1C). The most attenuation of brain infarction and neurological dysfunction by OA administration was observed at doses 10 and $30 \mathrm{mg} / \mathrm{kg}$ without any statistical difference between them (Fig. 1B, 1C). Therefore, $\mathrm{OA}$ at $10 \mathrm{mg} / \mathrm{kg}$ was used for further experiments. Next, we evaluated whether the neuroprotection could extend the time window by assessing the effects of delayed OA administration ( $3 \mathrm{~h}$ after reperfusion). After delayed OA administration, brain infarction and neurological dysfunction during the acute phase ( 1 day after tMCAO challenge) were significantly attenuated as compared with the vehicle-administered group (Fig. 1D-1F). These results indicate that a therapeutic regimen of OA can provide neuroprotection against acute brain injury following ischemic stroke.

During the ischemic stroke acute phase, most of the cells, including neurons, in the ischemic core region can undergo apoptosis (MacManus and Buchan, 2000; Sairanen et al., 2006). Therefore, we evaluated neuronal apoptosis using TUNEL assay 1 day after IMCAO challenge. In the vehicletreated mice group, many neurons underwent apoptosis in the ischemic core region (Fig. 2), while OA administration significantly reduced neuronal apoptosis (Fig. 2). These results indicate that OA can attenuate the IMCAO-induced neuronal apoptosis in injured brains.

\section{$O A$ attenuates microglial activation and lipid peroxidation in injured brains after TMCAO challenge}

Microglial activation is a key pathogenic factor in acute brain injury after the ischemic stroke (Yenari et al., 2010). Previously, it has been also reported that OA can attenuate microglial activation and production of inflammatory cytokines in LPS-stimulated BV2 microglia in vitro (Castellano et al., 2019). Therefore, OA neuroprotection observed during the acute phase could be associated with attenuation of microglial activation in an injured brain after tMCAO challenge. We verified this by lba1 immunohistochemical analysis in different regions (in periischemic and ischemic core regions) and different time points ( 1 and 3 days after tMCAO challenge). In the vehicle-treated mice, the number of Iba1-positive cells was markedly increased in the periischemic and ischemic core regions at both time points ( 1 day, Fig. 3A, 3B; 3 days, Fig. 3C, 3D). OA administration significantly reduced the number of activated microglia at both regions and both time points (Fig. 3A-3D). Another key characteristic feature of microglial activation after ischemic stroke challenge is a morphological change of microglia from ramified to amoeboid. In the present study, morphological changes can be clearly observed in the ischemic core region 3 days after the tMCAO challenge (Gaire et al., 2020; Sapkota et al., 2020); OA administration significantly reduced the number of amoeboid microglia (Fig. 3E). These 
A

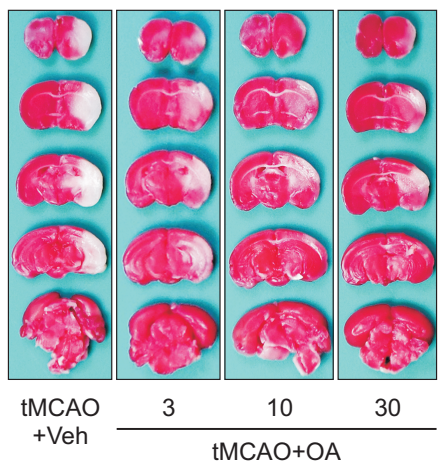

B

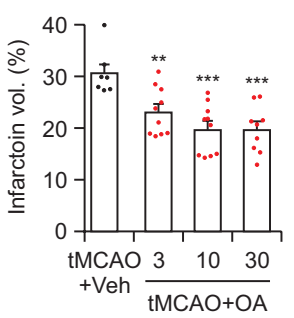

C

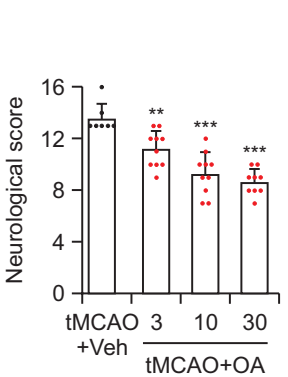

D

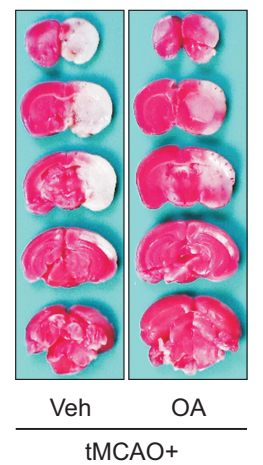

E

$\mathbf{F}$

Fig. 1. OA administration attenuates acute brain injury after $\mathrm{MCAO}$ challenge. Mice were challenged with $\mathrm{tMCAO}$. OA was administered immediately after reperfusion (A-C) or $3 \mathrm{~h}$ after reperfusion (D-F). (A-C) Effects of OA at different dosages (3, 10, and $30 \mathrm{mg} / \mathrm{kg}$; p.o.) on infraction volume $(A, B)$ and functional neurological deficits $(C)$ were measured 1 day after tMCAO. Representative images of TTC-stained brain sections (A) and quantification of brain infarction volume (B). Neurological score indicating mNSS (C). $n=7$ (tMCAO+Veh), 10 (tMCAO+OA (3 and $10 \mathrm{mg} / \mathrm{kg})$ ) and 9 (tMCAO+OA (30 mg/kg)). (D-F) Effects of delayed OA administration (10 mg/kg, p.o., at $3 \mathrm{~h}$ after reperfusion) on infraction volume (D, E) and functional neurological deficits $(F)$ were measured 1 day after tMCAO. Representative images of TTC-stained brain sections (D) and quantification of brain infarction volume (E). Neurological score $(F) . n=10$ mice per group. ${ }^{* *} p<0.01$ and ${ }^{* * *} p<0.001$ versus vehicle-administered tMCAO group.

A

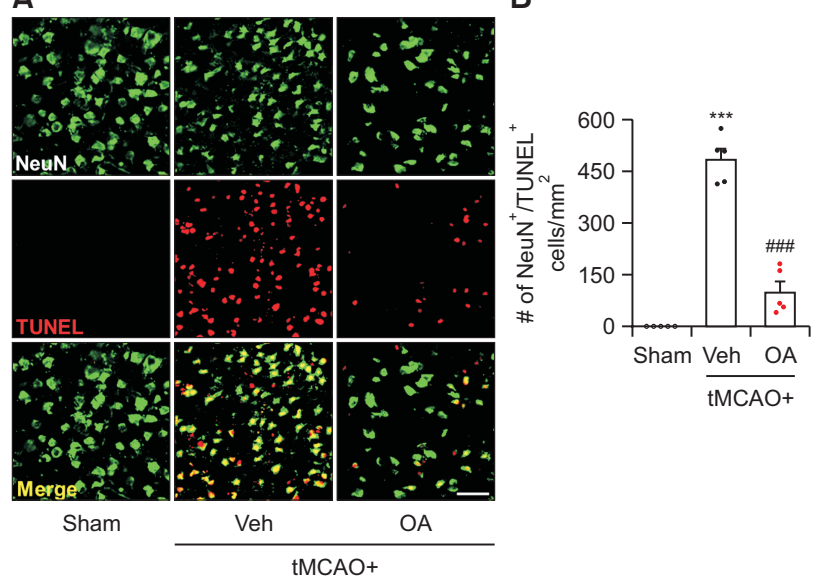

Fig. 2. OA administration attenuates neuronal apoptosis during the acute phase after tMCAO challenge. Mice were challenged with $\mathrm{tMCAO}$. OA (10 $\mathrm{mg} / \mathrm{kg}$, p.o.) was administered immediately after reperfusion. Effects of OA on neuronal apoptosis were assessed by TUNEL assay 1 day after the tMCAO challenge. Representative images of TUNEL/NeuN-double positive cells in the ischemic core region (A) and quantification (B). Scale bar, $50 \mu \mathrm{m} . \mathrm{n}=5$ mice per group. ${ }^{* * *} p<0.001$ versus sham; ${ }^{\# \#} p<0.001$ vs. vehicleadministered tMCAO group.

results indicate that $\mathrm{OA}$ can attenuate microglial activation in injured brains after ischemic stroke challenge.

Lipid peroxidation by oxidative stress is another important pathogenic factor in acute brain injury after ischemic stroke challenge (Muralikrishna Adibhatla and Hatcher, 2006). OA can reduce oxidative stress in many CNS diseases (Castellano et al., 2019; Gudoityte et al., 2021). Moreover, previous reports have demonstrated that a prophylactic regimen of OA could attenuate oxidative stress in injured brains after ischemic stroke challenge (Rong et al., 2011; Shi et al., 2021). In the present study, we evaluated whether a therapeutic regi- men of OA could attenuate lipid peroxidation in injured brains after tMCAO challenge by 4-HNE immunohistochemical analysis 1 and 3 days after the tMCAO challenge. In the vehicletreated mice, the number of 4-HNE-positive cells was dramatically increased in the ischemic core region at both day 1 (Fig. 4A, 4B) and day 3 (Fig. 4C, 4D) after the tMCAO challenge. However, $O A$ administration significantly reduced the number of 4-HNE-positive cells at both time points (Fig. 4A-4D). These data indicate that $O A$ can attenuate oxidative stress in injured brains after ischemic challenge.

\section{OA attenuates NLRP3 inflammasome activation in injured brains after $\mathrm{TMCAO}$ challenge}

NLRP3 inflammasome activation that involves NLRP3 priming and assembly of NLRP3 inflammasome (He et al., 2016; Kelley et al., 2019) is associated with acute brain injury after the ischemic stroke through increasing neuroinflammation (Lenart et al., 2016). NLRP3 priming can occur mostly in Iba1-immunopositive cells of injured brains after ischemic challenge, as evidenced by NLRP3 upregulation in these cells (Abulafia et al., 2009; Lee et al., 2020). Moreover, such upregulation can be clearly observed in the ischemic core region 3 days after the tMCAO challenge (Lee et al., 2020). Therefore, we evaluated whether OA could attenuate NLRP3 upregulation in Iba1-positive cells in the ischemic core region 3 days after tMCAO challenge by NLRP3/lba1 double immunofluorescence. In the vehicle-treated mice, the number of NLRP3/ Iba1-double immunopositive cells in the ischemic core region was markedly increased (Fig. 5A, 5B), while, in the OA administered group, the number of cells was significantly reduced (Fig. 5A, 5B). These results indicate that OA can attenuate NLRP3 priming in injured brains after ischemic stroke challenge.

We also evaluated whether OA could attenuate the assembly of NLRP3 inflammasome during the acute phase of ischemic stroke injury. We analyzed ASC speck formation, a critical feature of NLRP3 inflammasome assembly (He et al., 2016; Kelley et al., 2019), in the ischemic core region 3 days after the tMCAO challenge through NLRP3/ASC double im- 
A

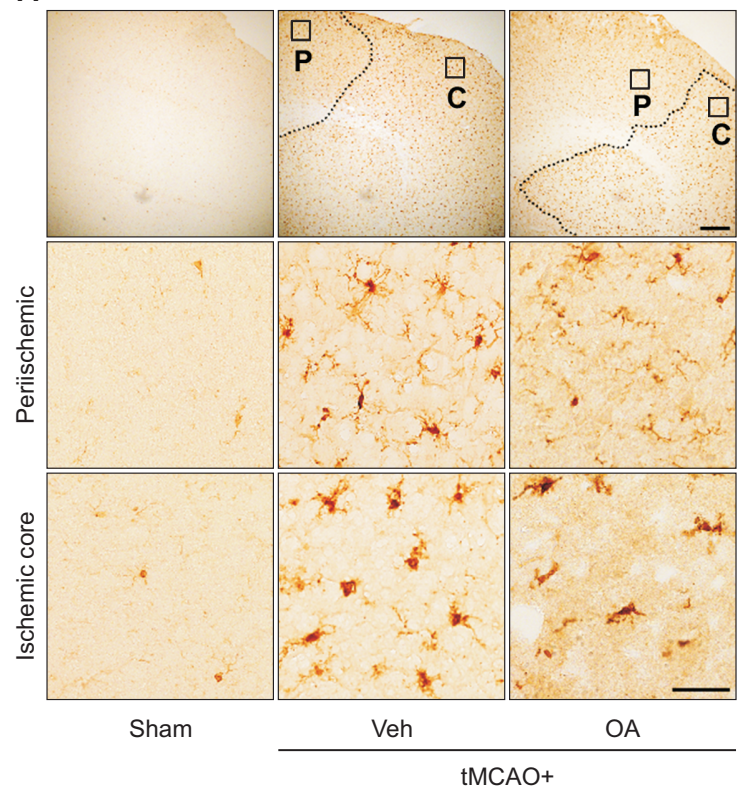

C

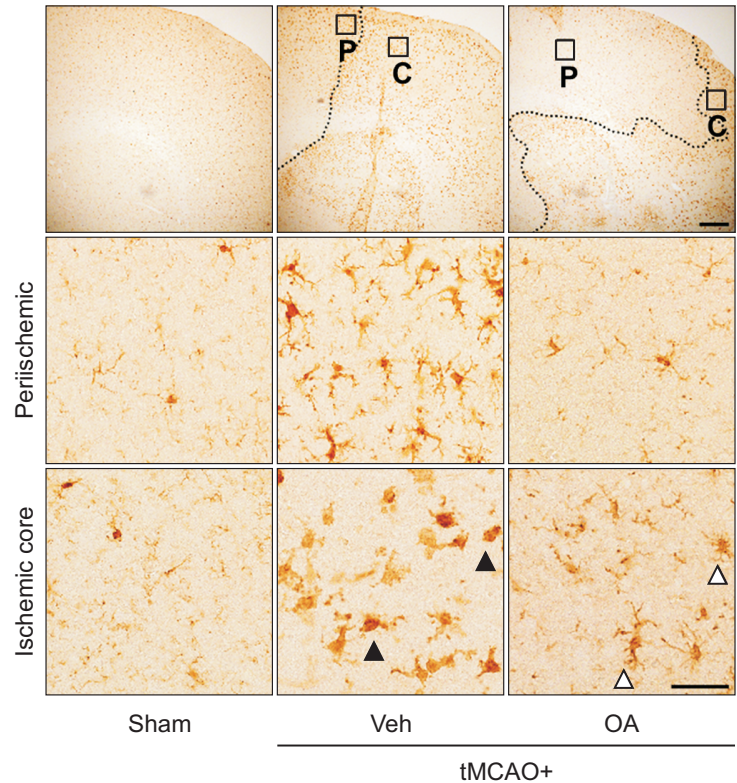

B

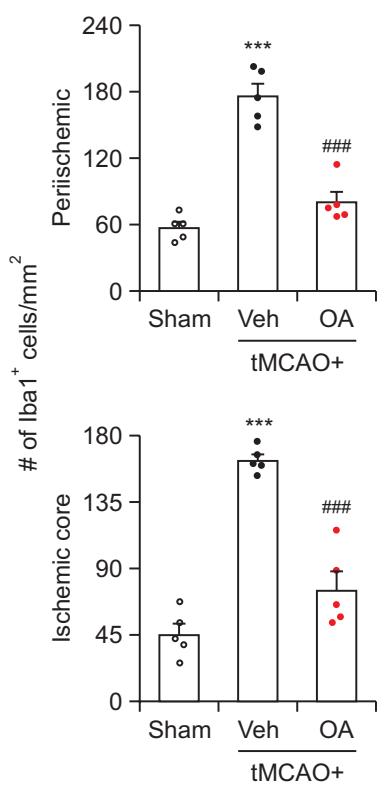

D

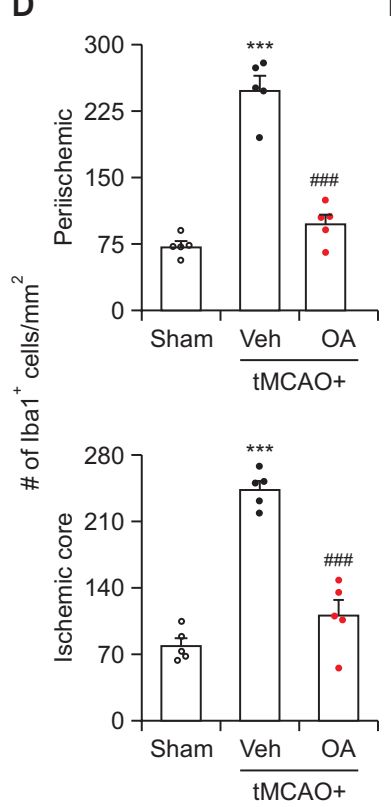

$\mathrm{E}$

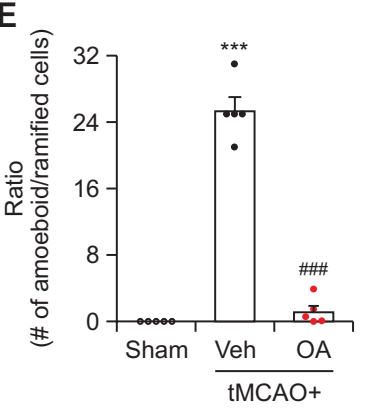

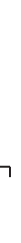

Fig. 3. OA administration attenuates microglial activation during the acute phase after $\mathrm{TMCAO}$ challenge. Mice were challenged with tMCAO. OA (10 mg/kg, p.o.) was administered immediately after reperfusion. (A, B) Effects of OA on microglial activation were determined by Iba1 immunohistochemistry 1 day after the tMCAO challenge. Representative images of Iba1-immunopositive cells in the periischemic ("P") and ischemic core ("C") regions (A) and quantification (B). Dotted lines separate the periischemic and ischemic core regions. (C-E) Effects of $\mathrm{OA}$ on microglial activation were determined 3 days after tMCAO challenge. Representative images of Iba1-immunopositive cells (C) and quantification (D) in each region. The ratio of amoeboid to ramified Iba1-immunopositive cells in the ischemic core region (E). Open and closed arrowheads (in C) represent ramified and amoeboid lba1-immunopositive cells, respectively. Scale bars, $200 \mu \mathrm{m}$ (top) and $50 \mu \mathrm{m}$ (middle and bottom). $\mathrm{n}=5$ mice per group. ${ }^{* * *} p<0.001$ versus sham; ${ }^{\# \#} p<0.001$ versus vehicle-administered tMCAO group.

munofluorescence. In the vehicle-treated mice, the number of NLRP3/ASC-double positive cells was markedly increased (Fig. 5C, 5D), while the number was significantly reduced in the OA administered group (Fig. 5C, 5D). These results indicate that OA can attenuate the assembly of NLRP3 inflammasome in injured brains after ischemic stroke.
OA attenuates chronic brain injury after tMCAO challenge

To evaluate OA neuroprotective effects against chronic brain injury after the tMCAO challenge, we analyzed mNSS up to 21 days and brain tissue loss at the end of the experiment. OA (10 mg/kg) was administered to mice every day for 7 days starting either immediately after reperfusion (immediate administration group) or 1 day after reperfusion (delayed ad- 
A

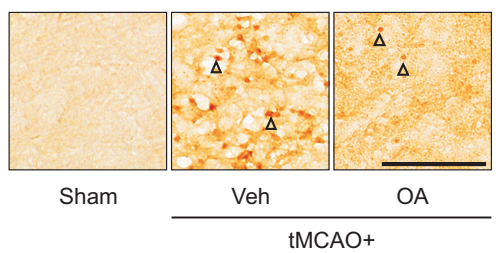

C

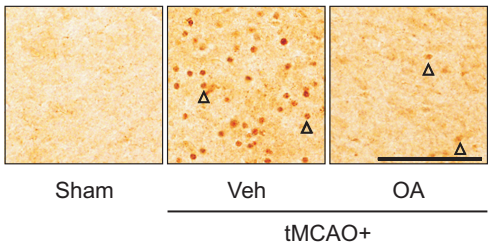

B

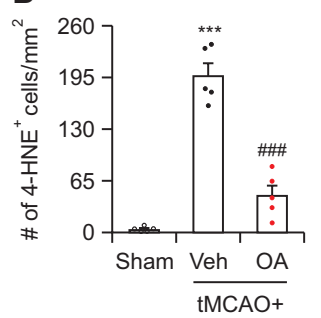

D

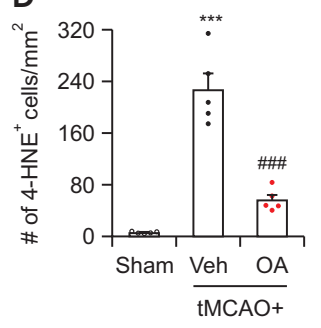

Fig. 4. OA administration attenuates lipid peroxidation during the acute phase after $\mathrm{MCAO}$ challenge. Mice were challenged with tMCAO. OA $(10 \mathrm{mg} / \mathrm{kg}$, p.o.) was administered immediately after reperfusion. (A, B) Effects of $O A$ on lipid peroxidation were determined by 4-HNE immunohistochemistry 1 day after the tMCAO challenge. Representative images of 4-HNE-immunopositive cells in the ischemic core region $(A)$ and quantification $(B)$. (C, D) Effects of $O A$ on lipid peroxidation were determined 3 days after the tMCAO challenge. Representative images of 4-HNE-immunopositive cells in the ischemic core region (C) and quantification (D). Arrowheads indicate 4 -HNE-immunopositive cells. Scale bars, 50 $\mu \mathrm{m}$. $\mathrm{n}=5$ mice per group. ${ }^{* *} p<0.001$ versus sham; ${ }^{\# \#} p<0.001$ vs. vehicle-administered tMCAO group.

ministration group). Functional neurological deficits were significantly attenuated in the immediate administration and the delayed administration groups as compared with the vehicle administration group (Fig. 6A). There were more improvements of the neurological functions in the immediate administration group than in the delayed administration group at all determined time points except 3 days (Fig. 6A). However, the difference was not statistically significant. Next, we evaluated whether OA could attenuate brain atrophy after tMCAO challenge by measuring brain tissue loss at the end of the experiment ( 21 days after tMCAO challenge). Both the immediate and delayed $O A$ administration significantly attenuated the tMCAO-induced brain tissue loss as compared with the vehicle administration (Fig. 6B, 6C). These results indicate that $\mathrm{OA}$ can attenuate chronic brain injury after tMCAO challenge.

\section{DISCUSSION}

Ischemic stroke in the brain occurs by the interruption of cerebral blood flow and results in brain damage via various pathogenesis such as excitotoxicity, inflammation, and oxidative stress (Jayaraj et al., 2019). Tissue plasminogen activator, a thrombolytic agent, is widely used to treat acute ischemic stroke despite its limited time window efficacy (within $4.5 \mathrm{~h}$ after stroke onset) and side effects (Pu et al., 2019). Edaravone, a free radical scavenger, is also in use only in Japan for the same purpose (Lapchak, 2010). Besides these drugs, numerous neuroprotectants for ischemic stroke have

been developed through preclinical experiments ( $\mathrm{O}^{\prime} \mathrm{Collins}$ et al., 2006), but unfortunately no agent has been successful in clinical translation (Gladstone et al., 2002; Schmidt-Pogoda et al., 2020). This might be due to, in part, an insufficient efficacy validation (Gladstone et al., 2002; Schmidt-Pogoda et al., 2020). To develop efficient neuroprotectants, the efficacy must be validated in consideration of several issues such as therapeutic effects of tested drugs rather than prophylactic effects, time window efficacy, and long-term neuroprotection (Gladstone et al., 2002; Schmidt-Pogoda et al., 2020). In this regard, the present study verified OA effects as a promising neuroprotectant for ischemic stroke, along with underlying mechanisms of action. Our experiment results evidenced that OA administration immediately after reperfusion reduced brain damage in IMCAO-challenged mice during both acute and chronic phases, suggesting that it is a potential therapeutic agent for ischemic stroke. The therapeutic effects were observed even with delayed OA administration ( $3 \mathrm{~h}$ for the acute brain injury and 1 day for the chronic brain injury). In view of acute brain injury-relevant pathogenesis, OA could attenuate microglial activation and lipid peroxidation in the injured brains. Moreover, OA suppressed NLRP inflammasome activation by attenuating both NLRP3 upregulation and assembly of NLRP3 inflammasome in the injured brains.

In previous studies, OA was demonstrated to exert beneficial effects against acute brain injury following ischemic stroke using in vitro (Gu, 2021) and in vivo models (Rong et al., 2011; Shi et al., 2021). OA can attenuate the death of PC12 cells subjected to oxygen/glucose deprivation (Gu, 2021). Further, OA can also attenuate brain damage, including brain infarction and functional neurological deficits during the acute phase after tMCAO challenge (Rong et al., 2011; Shi et al., 2021). Similarly, we observed that OA could attenuate acute brain damage after tMCAO challenge. However, our present study further demonstrated novel findings on the therapeutic efficacy of OA against acute brain injury following ischemic stroke. The present study has shown that OA administration immediately after IMCAO challenge can attenuate such brain damage, while previous studies showed that OA administration prior to tMCAO can prevent brain damage (Rong et al., 2011; Shi et al., 2021). Importantly, the time-window experiment in the present study showed that delayed administration of OA (i.e., $3 \mathrm{~h}$ after reperfusion) can also attenuate acute brain damage, supporting therapeutic effects of OA during the acute phase after ischemic stroke. Besides effects against ischemic stroke-induced acute brain injury, we demonstrated that OA can attenuate chronic brain injury of ischemic stroke; daily administration of OA within a short period (7 days) after tMCAO challenge also attenuated long-term neurological dysfunction up to 21 days after the challenge and tissue loss at the end of experiments. These results are consistent with a previous study (Shi et al., 2021) which demonstrated that daily administration of OA for 6 weeks after IMCAO challenge can attenuate neurological dysfunction up to 7 weeks after the challenge and brain tissue loss at the end of experiments (Shi et al., 2021). Importantly, the present study findings revealed that the longterm neuroprotective effects of OA could also be observed with delayed administration of OA (daily administration for 7 days starting at 1 day after tMCAO challenge). These current and previous studies (Shi et al., 2021) have clearly suggested that $O A$ is also neuroprotective against chronic brain injury after IMCAO challenge. Considering that long-term neurological 
A

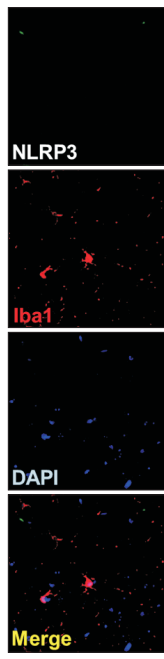

Sham
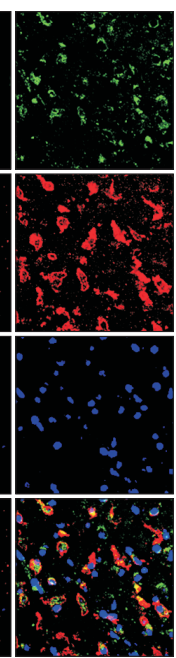

Veh
B

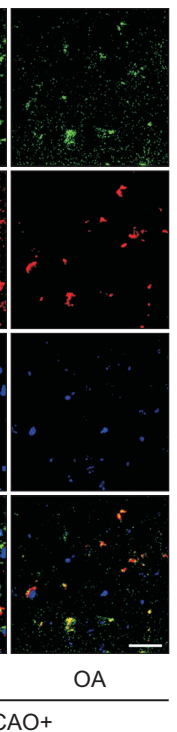

C

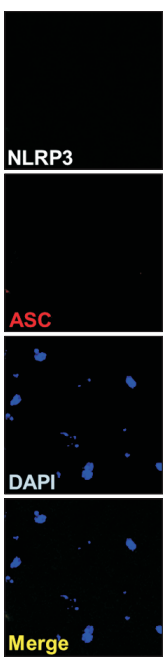

Sham

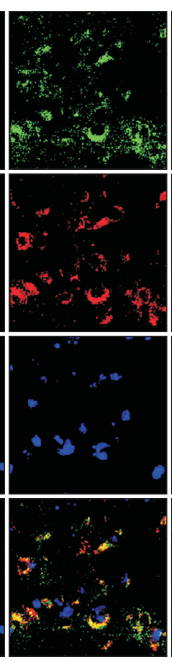

Veh

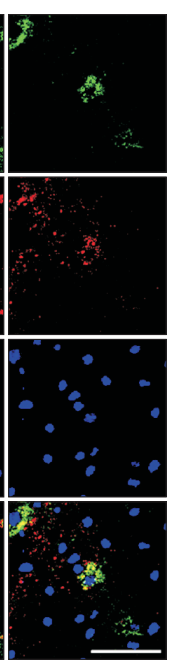

OA

tMCAO+

D

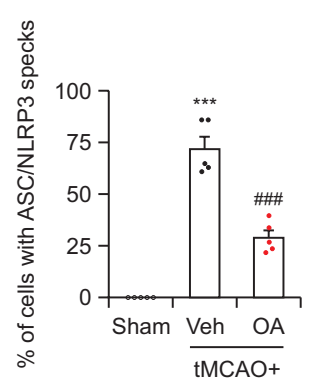

Fig. 5. OA administration attenuates NLRP3 inflammasome activation during the acute phase after tMCAO challenge. Mice were challenged with tMCAO. OA (10 mg/kg, p.o.) was administered immediately after reperfusion. (A, B) Effects of OA on NLRP3 upregulation in Iba1-immunopositive cells were assessed by NLRP3/lba1 double immunofluorescence 3 days after the tMCAO challenge. Representative images of NLRP3/lba1-double immunopositive cells in the ischemic core region (A) and quantification (B). (C, D) Effects of OA on ASC speck formation in NLRP3-immunopositive cells were assessed by ASC/NLRP3 double immunofluorescence 3 days after the tMCAO challenge. Representative images of ASC/NLRP3-double immunopositive cells in the ischemic core region (C) and quantification (D). Scale bars, $50 \mu \mathrm{m} . \mathrm{n}=5$ mice per group. ${ }^{* * *} p<0.001$ versus sham; ${ }^{\# \#} p<0.001$ vs. vehicle-administered tMCAO group.

A

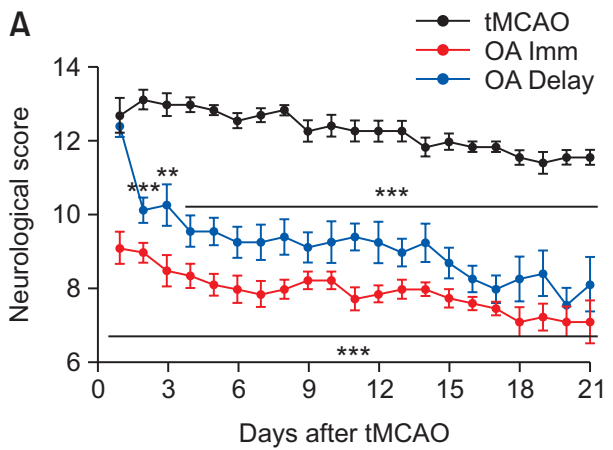

B

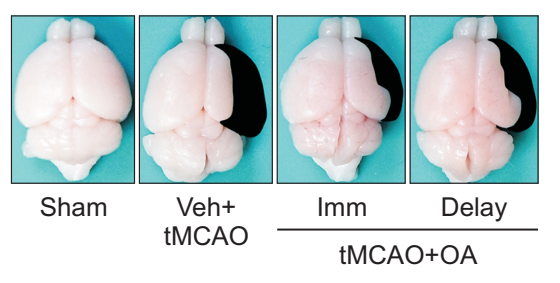

C

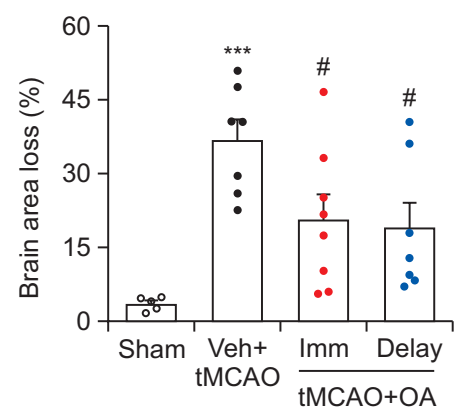

Fig. 6. OA administration attenuates chronic brain injury after $\mathrm{tMCAO}$ challenge. Mice were challenged with $\mathrm{tMCAO}$. OA (10 mg/kg, p.o.) was administered to mice once per day for 7 days starting either immediately after reperfusion (immediate administration group, "Imm") or 1 day after reperfusion (delayed administration group, "Delay"). (A) Effects of OA on functional neurological deficits were determined for up to 21 days after $\mathrm{tMCAO}$ challenge. $\mathrm{n}=7$ ( $\mathrm{tMCAO}+\mathrm{Veh}$, black solid line), 8 ( $\mathrm{MCAO}+\mathrm{mm}$, red solid line), and 7 (tMCAO+Delay, blue solid line). ${ }^{* *} p<0.01$ and ${ }^{* * *} p<0.001$ versus vehicle-administered tMCAO group. $(B, C)$ Effects of OA on brain tissue loss were determined 21 days after tMCAO challenge. Representative images of brain tissue loss $(B)$ and quantification $(C)$. Black colored area (in $B$ ) indicate lost area. $n=5$ for

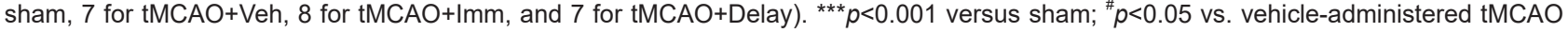
group.

disability is a major challenge for ischemic stroke survivors (Girard et al., 2014), based on the present and previous findings, OA could be an effective therapeutic option for ischemic stroke patients.

Emerging evidence has suggested that neuroinflammation plays an important role in the pathogenesis of ischemic stroke and is closely associated with microglial activation (Shah et al., 2009; Jin et al., 2010; Xu et al., 2020). Therefore, suppressing microglial activation could be an effective basis for the drug development of an ischemic stroke therapeutic agent. OA has been shown to exert anti-inflammatory activities in vivo through several mechanisms such as reducing microglial activation in different CNS diseases (Martin et al., 2012; Castellano et al., 2019; Msibi and Mabandla, 2019). For instance, OA significantly reduced microglial activation and their inflammatory responses in vitro in LPS-stimulated BV2 microglial cells (Castellano et al., 2019). The present study clearly demonstrated that OA administration significantly reduced the number of activated microglia (Iba1-immunopositive cells) and reversed their morphological changes from 
toxic amoeboid cells to less toxic ramified cells in the ischemic core and periischemic regions of the injured brains during the acute phase. These results are consistent with those of a previous study that reported that OA reduces the number of amoeboid-shaped microglia in the injured hippocampus after tMCAO challenge during the chronic phase (Shi et al., 2021). These independent findings indicate that the neuroprotective effects of OA against both acute and chronic brain injuries following ischemic stroke are associated with attenuation of microglial activation.

Oxidative stress is another key factor in the pathogenesis of ischemic stroke, leading to lipid peroxidation, cell death, and inflammatory responses (Muralikrishna Adibhatla and Hatcher, 2006). In a previous study, OA has been shown to suppress oxidative stress in several CNS diseases (Gudoityte et al., 2021). Similarly, in ischemic stroke, pre-administration of OA prior to tMCAO challenge was shown to increase activities of endogenous antioxidants, such as superoxide dismutase and glutathione peroxidase, and reduce malondialdehyde levels in injured brains following ischemic stroke (Rong et al., 2011; Shi et al., 2021). In the present study, similar OA anti-oxidative activities were demonstrated in which $\mathrm{OA}$ administration after reperfusion reduced $\mathrm{IMCAO}$-induced lipid peroxidation in the ischemic core region. These results suggest that neuroprotective effects of both prophylactic and therapeutic OA regimens are associated with the suppression of oxidative stress.

NLRP3 inflammasome activation that is characterized by the sequential processing of NLRP3 upregulation and assembly of inflammasome components such as NLRP3, ASC, caspase-1, and IL-1 $\beta$ (He et al., 2016; Kelley et al., 2019) has been suggested as a key pathogenic event in ischemic stroke (Yang et al., 2014; Ye et al., 2017; Ismael et al., 2018; Lee et al., 2020). Indeed, genetic deletion of NLRP3 or pharmacological suppression of NLRP3 inflammasome can reduce ischemic stroke-induced acute brain injury (Yang et al., 2014; Ye et al., 2017; Ismael et al., 2018). OA has also been reported to attenuate NLRP3 inflammasome activation in several diseases including diabetic complications and lung fibrosis (An et al., 2017; Kim et al., 2018). The current study suggested ischemic stroke as a target for beneficial pharmacological effects of OA; it was shown to attenuate NLRP3 inflammasome activation in injured brains after IMCAO challenge during the acute phase by suppressing NLRP3 upregulation and inhibiting ASC speck formation, an essential step for the assembly of NLRP3 inflammasome. Moreover, similar attenuation by OA was observed in activated microglia, a key cell type for NLRP3 inflammasome activation in ischemic stroke-induced brain injury (Yang et al., 2014; Lee et al., 2020). Notably, NLRP3 inflammasome activation can occur in activated microglia (Abulafia et al., 2009; Lee et al., 2020) and can be driven by oxidative stress after ischemic stroke (Minutoli et al., 2016; $\mathrm{Xu}$ et al., 2021). Considering that OA can attenuate microglial activation and oxidative stress as demonstrated by our findings, suppressed NLRP3 inflammasome activation may be an underlying molecular mechanism of neuroprotective effects of OA against ischemic stroke.

In conclusion, the present study demonstrated that a therapeutic regimen of OA could attenuate ischemic stroke-induced acute and chronic brain injuries. The neuroprotective effects of OA against ischemic stroke-induced acute brain injury were associated with attenuated neuroinflammation, including microglial activation, oxidative stress, and NLRP3 inflamma- some activation. In addition, the present study findings indicate that naturally occurring $O A$ can be a potential therapeutic agent for ischemic stroke. Moreover, considering its pharmacological potential to attenuate neuroinflammation, OA could be a therapeutic agent for other inflammatory CNS diseases.

\section{CONFLICT OF INTEREST}

The authors declare no conflict of interests.

\section{ACKNOWLEDGMENTS}

This work was supported by grants from the National Research Foundation (NRF) funded by the Korean government to JWC [NRF-2020M3A9E4104384, NRF2020R1A6A1A03043708, and NRF-2021R1A2C1005520]. We thank $\mathrm{CH}$ Lee for providing assistance for a blind determination.

\section{REFERENCES}

Abulafia, D. P., de Rivero Vaccari, J. P., Lozano, J. D., Lotocki, G., Keane, R. W. and Dietrich, W. D. (2009) Inhibition of the inflammasome complex reduces the inflammatory response after thromboembolic stroke in mice. J. Cereb. Blood Flow Metab. 29, 534-544.

An, Q., Hu, Q., Wang, B., Cui, W., Wu, F. and Ding, Y. (2017) Oleanolic acid alleviates diabetic rat carotid artery injury through the inhibition of NLRP3 inflammasome signaling pathways. Mol. Med. Rep. 16, 8413-8419.

Ayeleso, T. B., Matumba, M. G. and Mukwevho, E. (2017) Oleanolic acid and its derivatives: biological activities and therapeutic potential in chronic diseases. Molecules 22, 1915.

Caltana, L., Rutolo, D., Nieto, M. L. and Brusco, A. (2014) Further evidence for the neuroprotective role of oleanolic acid in a model of focal brain hypoxia in rats. Neurochem. Int. 79, 79-87.

Castellano, J. M., Garcia-Rodriguez, S., Espinosa, J. M., Millan-Linares, M. C., Rada, M. and Perona, J. S. (2019) Oleanolic acid exerts a neuroprotective effect against microglial cell activation by modulating cytokine release and antioxidant defense systems. Biomolecules 9,683 .

Castellano, J. M., Guinda, A., Delgado, T., Rada, M. and Cayuela, J. A. (2013) Biochemical basis of the antidiabetic activity of oleanolic acid and related pentacyclic triterpenes. Diabetes 62, 1791-1799.

Gaire, B. P., Sapkota, A. and Choi, J. W. (2020) BMS-986020, a specific LPA1 antagonist, provides neuroprotection against ischemic stroke in mice. Antioxidants 9, 1097.

Girard, S., Murray, K. N., Rothwell, N. J., Metz, G. A. and Allan, S. M. (2014) Long-term functional recovery and compensation after cerebral ischemia in rats. Behav. Brain. Res. 270, 18-28.

Gladstone, D. J., Black, S. E. and Hakim, A. M. (2002) Toward wisdom from failure: lessons from neuroprotective stroke trials and new therapeutic directions. Stroke 33, 2123-2136.

$\mathrm{Gu}$, S. (2021) Oleanolic acid improved inflammatory response and apoptosis of PC12 cells induced by OGD/R through downregulating miR-142-5P. Nat. Prod. Commun. 16, 1934578X211018019.

Gudoityte, E., Arandarcikaite, O., Mazeikiene, I., Bendokas, V. and Liobikas, J. (2021) Ursolic and oleanolic acids: plant metabolites with neuroprotective potential. Int. J. Mol. Sci. 22, 4599.

He, Y., Hara, H. and Nunez, G. (2016) Mechanism and regulation of NLRP3 inflammasome activation. Trends Biochem. Sci. 41, 10121021.

Ismael, S., Zhao, L., Nasoohi, S. and Ishrat, T. (2018) Inhibition of the NLRP3-inflammasome as a potential approach for neuroprotection after stroke. Sci. Rep. 8, 5971.

Jayaraj, R. L., Azimullah, S., Beiram, R., Jalal, F. Y. and Rosenberg, G. 
A. (2019) Neuroinflammation: friend and foe for ischemic stroke. J. Neuroinflammation 16, 142.

Jin, R., Yang, G. and Li, G. (2010) Inflammatory mechanisms in ischemic stroke: role of inflammatory cells. J. Leukoc. Biol. 87, 779789

Kelley, N., Jeltema, D., Duan, Y. and He, Y. (2019) The NLRP3 inflammasome: an overview of mechanisms of activation and regulation. Int. J. Mol. Sci. 20, 3328.

Kim, M. S., Han, J. Y., Kim, S. H., Jeon, D., Kim, H. Y., Lee, S. W., Rho, M. C. and Lee, K. (2018) Oleanolic acid acetate attenuates polyhexamethylene guanidine phosphate-induced pulmonary inflammation and fibrosis in mice. Respir. Physiol. Neurobiol. 252253, 1-9.

Lapchak, P. A. (2010) A critical assessment of edaravone acute ischemic stroke efficacy trials: is edaravone an effective neuroprotective therapy? Expert. Opin. Pharmacother. 11, 1753-1763.

Lee, C. H., Sapkota, A., Gaire, B. P. and Choi, J. W. (2020) NLRP3 Inflammasome activation is involved in LPA1-mediated brain injury after transient focal cerebral ischemia. Int. J. Mol. Sci. 21, 8595.

Lenart, N., Brough, D. and Denes, A. (2016) Inflammasomes link vascular disease with neuroinflammation and brain disorders. $J$. Cereb. Blood Flow. Metab. 36, 1668-1685.

Liu, J. (2005) Oleanolic acid and ursolic acid: research perspectives. J. Ethnopharmacol. 100, 92-94.

MacManus, J. P. and Buchan, A. M. (2000) Apoptosis after experimental stroke: fact or fashion? J. Neurotrauma 17, 899-914.

Martin, R., Carvalho-Tavares, J., Hernandez, M., Arnes, M., Ruiz-Gutierrez, V. and Nieto, M. L. (2010) Beneficial actions of oleanolic acid in an experimental model of multiple sclerosis: a potential therapeutic role. Biochem. Pharmacol. 79, 198-208.

Martin, R., Cordova, C., San Roman, J. A., Gutierrez, B., Cachofeiro, V. and Nieto, M. L. (2014) Oleanolic acid modulates the immune-inflammatory response in mice with experimental autoimmune myocarditis and protects from cardiac injury. Therapeutic implications for the human disease. J. Mol. Cell. Cardiol. 72, 250-262.

Martin, R., Hernandez, M., Cordova, C. and Nieto, M. L. (2012) Natural triterpenes modulate immune-inflammatory markers of experimental autoimmune encephalomyelitis: therapeutic implications for multiple sclerosis. Br. J. Pharmacol. 166, 1708-1723.

Minutoli, L., Puzzolo, D., Rinaldi, M., Irrera, N., Marini, H., Arcoraci, V., Bitto, A., Crea, G., Pisani, A., Squadrito, F., Trichilo, V., Bruschetta, D., Micali, A. and Altavilla, D. (2016) ROS-mediated NLRP3 inflammasome activation in brain, heart, kidney, and testis ischemia/reperfusion injury. Oxid. Med. Cell. Longev. 2016, 2183026.

Msibi, Z. N. P. and Mabandla, M. V. (2019) Oleanolic acid mitigates 6-hydroxydopamine neurotoxicity by attenuating intracellular ROS in PC12 cells and striatal microglial activation in rat brains. Front. Physiol. 10, 1059.

Muralikrishna Adibhatla, R. and Hatcher, J. F. (2006) Phospholipase $A 2$, reactive oxygen species, and lipid peroxidation in cerebral ischemia. Free Radic. Biol. Med. 40, 376-387.

Niu, G., Sun, L., Pei, Y. and Wang, D. (2018) Oleanolic acid inhibits colorectal cancer angiogenesis by blocking the VEGFR2 signaling pathway. Anticancer Agents Med. Chem. 18, 583-590.

O'Collins, V. E., Macleod, M. R., Donnan, G. A., Horky, L. L., van der Worp, B. H. and Howells, D. W. (2006) 1,026 experimental treatments in acute stroke. Ann. Neurol. 59, 467-477.

Pu, H., Shi, Y., Zhang, L., Lu, Z., Ye, Q., Leak, R. K., Xu, F., Ma, S., Mu, H., Wei, Z., Xu, N., Xia, Y., Hu, X., Hitchens, T. K., Bennett, M. V. L. and Chen, J. (2019) Protease-independent action of tissue plasminogen activator in brain plasticity and neurological recovery after ischemic stroke. Proc. Natl. Acad. Sci. U.S.A. 116, 9115-9124.
Rong, Z. T., Gong, X. J., Sun, H. B., Li, Y. M. and Ji, H. (2011) Protective effects of oleanolic acid on cerebral ischemic damage in vivo and $\mathrm{H}(2) \mathrm{O}(2)$-induced injury in vitro. Pharm. Biol. 49, 78-85.

Sairanen, T., Karjalainen-Lindsberg, M. L., Paetau, A., ljas, P. and Lindsberg, P. J. (2006) Apoptosis dominant in the periinfarct area of human ischaemic stroke--a possible target of antiapoptotic treatments. Brain 129, 189-199.

Sapkota, A., Lee, C. H., Park, S. J. and Choi, J. W. (2020) Lysophosphatidic acid receptor 5 plays a pathogenic role in brain damage after focal cerebral ischemia by modulating neuroinflammatory responses. Cells 9, 1446.

Schmidt-Pogoda, A., Bonberg, N., Koecke, M. H. M., Strecker, J. K., Wellmann, J., Bruckmann, N. M., Beuker, C., Schabitz, W. R., Meuth, S. G., Wiendl, H., Minnerup, H. and Minnerup, J. (2020) Why most acute stroke studies are positive in animals but not in patients: a systematic comparison of preclinical, early phase, and phase 3 clinical trials of neuroprotective agents. Ann. Neurol. 87, 40-51.

Shah, I. M., Macrae, I. M. and Di Napoli, M. (2009) Neuroinflammation and neuroprotective strategies in acute ischaemic stroke - from bench to bedside. Curr. Mol. Med. 9, 336-354.

Shi, Y. J., Sun, L. L., Ji, X., Shi, R., Xu, F. and Gu, J. H. (2021) Neuroprotective effects of oleanolic acid against cerebral ischemiareperfusion injury in mice. Exp. Neurol. 343, 113785.

Wang, J. L., Ren, C. H., Feng, J., Ou, C. H. and Liu, L. (2020) Oleanolic acid inhibits mouse spinal cord injury through suppressing inflammation and apoptosis via the blockage of p38 and JNK MAPKs. Biomed. Pharmacother. 123, 109752.

Wang, K., Sun, W., Zhang, L., Guo, W., Xu, J., Liu, S., Zhou, Z. and Zhang, Y. (2018a) Oleanolic acid ameliorates Abeta25-35 injectioninduced memory deficit in Alzheimer's disease model rats by maintaining synaptic plasticity. CNS Neurol. Disord. Drug Targets 17, 389-399.

Wang, W., Chen, K., Xia, Y., Mo, W., Wang, F., Dai, W. and Niu, P. (2018b) The hepatoprotection by oleanolic acid preconditioning: focusing on PPARalpha activation. PPAR Res. 2018, 3180396.

Xu, K., Chu, F., Li, G., Xu, X., Wang, P., Song, J., Zhou, S. and Lei, H. (2014) Oleanolic acid synthetic oligoglycosides: a review on recent progress in biological activities. Pharmazie 69, 483-495.

Xu, Q., Zhao, B., Ye, Y., Li, Y., Zhang, Y., Xiong, X. and Gu, L. (2021) Relevant mediators involved in and therapies targeting the inflammatory response induced by activation of the NLRP3 inflammasome in ischemic stroke. J. Neuroinflammation 18, 123.

Xu, S., Lu, J., Shao, A., Zhang, J. H. and Zhang, J. (2020) Glial cells: role of the immune response in ischemic stroke. Front. Immunol. 11, 294.

Yang, F., Wang, Z., Wei, X., Han, H., Meng, X., Zhang, Y., Shi, W., Li, F., Xin, T., Pang, Q. and Yi, F. (2014) NLRP3 deficiency ameliorates neurovascular damage in experimental ischemic stroke. J. Cereb. Blood Flow Metab. 34, 660-667.

Ye, X., Shen, T., Hu, J., Zhang, L., Zhang, Y., Bao, L., Cui, C., Jin, G., Zan, K., Zhang, Z., Yang, X., Shi, H., Zu, J., Yu, M., Song, C., Wang, Y., Qi, S. and Cui, G. (2017) Purinergic 2X7 receptor/NLRP3 pathway triggers neuronal apoptosis after ischemic stroke in the mouse. Exp. Neurol. 292, 46-55.

Yenari, M. A., Kauppinen, T. M. and Swanson, R. A. (2010) Microglial activation in stroke: therapeutic targets. Neurotherapeutics 7, 378391.

Ziberna, L., Samec, D., Mocan, A., Nabavi, S. F., Bishayee, A., Farooqi, A. A., Sureda, A. and Nabavi, S. M. (2017) Oleanolic acid alters multiple cell signaling pathways: implication in cancer prevention and therapy. Int. J. Mol. Sci. 18, 643. 